\title{
Photonic Architecture for Scalable Quantum Information Processing in Diamond
}

\author{
Kae Nemoto, ${ }^{1, *}$ Michael Trupke, ${ }^{2}$ Simon J. Devitt, ${ }^{1}$ Ashley M. Stephens, ${ }^{1}$ Burkhard Scharfenberger, ${ }^{1}$ Kathrin Buczak, ${ }^{2}$ \\ Tobias Nöbauer, ${ }^{2}$ Mark S. Everitt, ${ }^{1}$ Jörg Schmiedmayer, ${ }^{2}$ and William J. Munro ${ }^{3,1}$ \\ ${ }^{1}$ National Institute of Informatics, 2-1-2 Hitotsubashi, Chiyoda-ku, Tokyo 101-8430, Japan \\ ${ }^{2}$ Vienna Center for Quantum Science and Technology, Atominstitut, TU Wien, 1020 Vienna, Austria \\ ${ }^{3}$ NTT Basic Research Laboratories, NTT Corporation, 3-1 Morinosato-Wakamiya, \\ Atsugi 243-0198, Japan
}

(Received 17 December 2013; revised manuscript received 9 July 2014; published 4 August 2014)

\begin{abstract}
Physics and information are intimately connected, and the ultimate information processing devices will be those that harness the principles of quantum mechanics. Many physical systems have been identified as candidates for quantum information processing, but none of them are immune from errors. The challenge remains to find a path from the experiments of today to a reliable and scalable quantum computer. Here, we develop an architecture based on a simple module comprising an optical cavity containing a single negatively charged nitrogen vacancy center in diamond. Modules are connected by photons propagating in a fiber-optical network and collectively used to generate a topological cluster state, a robust substrate for quantum information processing. In principle, all processes in the architecture can be deterministic, but current limitations lead to processes that are probabilistic but heralded. We find that the architecture enables large-scale quantum information processing with existing technology.
\end{abstract}

DOI: 10.1103/PhysRevX.4.031022

\section{INTRODUCTION}

Quantum computers promise to surpass even the fastest classical computers, but the task of building a quantum computer presents a significant challenge. Even if they are precisely engineered, all known quantum systems suffer from decoherence and dephasing [1]. If this noise is sufficiently weak and not too strongly correlated, then it can be suppressed with quantum error correction [2]. The role of quantum computer architecture is to integrate quantum error correction with feasible experimental technology, to find a path to a reliable and scalable quantum computer. In this context, of the many physical systems identified as candidates for quantum information processing [1], the negatively charged nitrogen-vacancy $\left(\mathrm{NV}^{-}\right)$ center in diamond [3-5] features a number of desirable properties [6-12]. For example, the $\mathrm{NV}^{-}$center possesses both a nuclear spin and an electron spin-the nuclear spin can serve as a memory to store quantum information for relatively long times [13], and the electron spin can be coupled to a photon to serve as a flexible interface with other $\mathrm{NV}^{-}$centers [14]. The experimental feasibility of this system has been well established in recent years. Experiments have demonstrated individual electron and

\footnotetext{
nemoto@nii.ac.jp
}

Published by the American Physical Society under the terms of the Creative Commons Attribution 3.0 License. Further distribution of this work must maintain attribution to the author(s) and the published article's title, journal citation, and DOI.
Subject Areas: Condensed Matter Physics, Photonics, Quantum Information nuclear spin initialization, manipulation, and measurement [9,15-24], long-lived nuclear memories [13], a coherent interface between an electron spin and an optical field [14], entanglement between two remote electron spins [25], and optical cavities containing $\mathrm{NV}^{-}$centers [26-28]. Statedependent reflectivity has been demonstrated with atoms [29], though not yet with $\mathrm{NV}^{-}$centers. At the same time, new techniques for quantum error correction have lessened experimental requirements [30-32].

Here, we develop a quantum computer architecture based on a simple module comprising an optical cavity containing a single $\mathrm{NV}^{-}$center in diamond. Modules are connected by photons propagating in a fiber-optical network. The cavities mediate interactions between the photons and the electron spins, enabling entanglement distribution and readout. The electron spins are coupled to nuclear spins, which constitute long-lived quantum memories where quantum information is stored and processed. Aside from modules connected by optical fibers, other elements of the architecture are single-photon detection devices and classical control lines. These elements are laid out in a regular two-dimensional array, with sufficient connectivity between modules to enable topological cluster-state error correction [33-35]. This arrangement is independent of the size of the network. At a circuit level, we find the maximum tolerable error per elementary quantum gate to be approximately $0.73 \%$. However, by analyzing the architecture at the physical level, we also estimate how well each component of the module must operate for the system to meet this threshold and be truly 
scalable. The results of this analysis indicate that the architecture is consistent with present technology and might be achievable in the near future.

Quantum computer architectures have been proposed for several other solid-state systems, including superconducting qubits [36], electronic spins [37], and quantum dots [37-40]. Each of these physical systems has particular properties, which requires architectures to be tailored. For instance, quantum dots do not generally have nuclear spins that can be used as quantum memories, so an alternative approach must be considered [41]. Regardless, the electron spin entanglement distribution schemes used in our approach can be applied to quantum dots and those already proposed in quantum dots can be applied to our case [42-50].

\section{FUNDAMENTAL BUILDING BLOCKS}

Our approach can be adapted to a variety of promising physical systems, such as ions, neutral atoms, and quantum dots [51-55], and for this reason, we begin with a general description of the fundamental module. However, to show that the module can form the basis of a truly scalable architecture, we focus on a concrete implementation using $\mathrm{NV}^{-}$centers.

We begin our description of the architecture with an entanglement scheme based on the state-dependent reflectivity of a module consisting of an atom-cavity system $[44,46,56]$, as depicted in Fig. 1 . We can describe the emitter as a four-level system with transitions $|0\rangle \rightarrow\left|0_{E}\right\rangle$ and $|1\rangle \rightarrow\left|1_{E}\right\rangle$, each with a frequency $\omega_{0}$ and $\omega_{1}=\omega_{0}+\delta$, respectively. The probability for a photon to be reflected by a module with cooperativity $C$ and the cavity tuned to the interrogation frequency $\omega$ is given by [57]

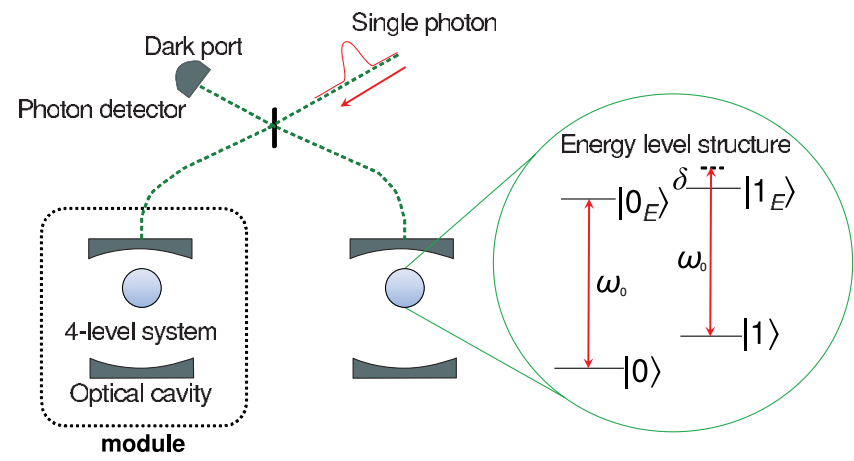

FIG. 1. Schematic representation illustrating the module and the entanglement distribution scheme. The module contains an optical cavity with a four-level system. The entanglement distribution scheme is based on a Michelson interferometer where two modules are connected via an optical fiber $[7,8,44,46]$. A single photon comes in from the right port and is conditionally reflected at each module depending on the state of the emitter. Erasing the path information at the beam splitter followed by detection at the dark port projects the system to the singlet Bell state.

$$
P_{R}=1-\frac{1+4 C+(\delta / \gamma)^{2}}{1+4 C+4 C^{2}+(\delta / \gamma)^{2}}
$$

Here, $\gamma$ is the amplitude decay rate of the interrogated transition, while $\delta=\omega_{1}-\omega_{0}$ is the detuning of cavity and interrogation light from that transition. We assume a cavity with matched mirrors, in which case an impinging photon will be reflected by the module with high probability if the emitter is in the ground state $|0\rangle$ and the cooperativity is $C \gg 1$. In the case of large detuning, $(\delta / \gamma)^{2} \gg\left(C^{2}, C, 1\right)$, the cavity is effectively empty and the reflection probability approaches $P_{R} \rightarrow 0$. In the simplest variant of our entanglement scheme (Fig. 1), we place two such modules at the output ports of a 50:50 beam splitter and prepare each emitter in an equal superposition of the ground states $|0\rangle$ and $|1\rangle$. A single photon is then sent onto the beam splitter. If it is subsequently detected at the "dark" port of the beam splitter, the emitters are projected onto the maximally entangled state

$$
|S\rangle=\frac{1}{\sqrt{2}}(|1\rangle|0\rangle-|0\rangle|1\rangle),
$$

with success probability $p=\eta^{2} P_{R} / 8$, where the collection efficiency $\eta^{2}$ includes the effects of inefficient sources and detectors (we provide full derails in the Supplemental Material [58] and also refer the interested reader to a textbook discussion of a similar scheme [56]). This probability may appear to be low; however, the generated entangled state has extremely high fidelity (>99\%) and is robust to imperfections (see Supplemental Material [58]). For instance, imbalance in the cavity reflection coefficients slightly reduces the success probability but does not degrade the fidelity of the resulting state.

The low success probability of the implementation can be simply overcome using a repeat-until-success approach to establish an entanglement link with high probability $[45,59]$. We show in the following sections that the scheme not only exhibits high fidelity in the presence of physical imperfections, but also, unlike other approaches, does not involve any catastrophic errors.

In addition, the module enables (near) perfect nondemolition measurement of the qubit state. For an architecture for quantum computation, we require a second qubit in the cavity to act as a quantum memory. Ideally, the coupling between our four-level system and this memory qubit can be switched on and off as required. This allows the four-level system to be reused for entanglement creation, now with a third module. By repeating this process with additional modules, we can generate a cluster state suitable for fault-tolerant quantum computation. In the following, we detail this architecture by describing a full implementation using single $\mathrm{NV}^{-}$centers in microcavities connected in a photonic network. 


\section{DIAMOND MODULE}

We now turn our attention to a concrete implementation: a fiber-connected optical cavity containing a single $\mathrm{NV}^{-}$ center, of which the energy levels are depicted in Fig. 2(a). The lowest three electron spin states, $\left|m_{s}=0, \pm 1\right\rangle \equiv$ $|0, \pm 1\rangle$ form the spin- ${ }^{3} A_{2}$ manifold, which has a zerofield splitting of $2.87 \mathrm{GHz}$. With an externally applied magnetic field $B_{z} \sim 20 \mathrm{mT}$ along the $\mathrm{NV}^{-}$axis (this suppresses non-spin-preserving spin transitions that appear for magnetic fields with components perpendicular to the $\mathrm{NV}^{-}$axis), our electron spin qubit levels $|0\rangle$ and $|+1\rangle$ are far detuned from the $|-1\rangle$ energy level and thus form an excellent qubit. The isotope ${ }^{15} \mathrm{~N}$ will be utilized as a spin$1 / 2$ nuclear memory [60]. Next, the optical transitions between one of the ${ }^{3} A_{2}$ magnetic sublevels $|i\rangle$ and the ${ }^{3} E$ levels $\left|M_{i}\right\rangle$ coupled to the cavity field can be represented by $\hbar g_{m_{s}, i} \sum_{i=1, \ldots, 6}\left[a^{\dagger}|i\rangle\left\langle M_{i}|+a| M_{i}\right\rangle\langle i|\right]$, where $g_{m_{s}, i}$ are the coupling constants between the transitions and field, $a^{\dagger}(a)$ are the field's creation (annihilation) operators and $M_{i}$ are the energy eigenstates, in order of ascending energy, within the ${ }^{3} E$ manifold. These transitions are fully resolved at temperature below $8 \mathrm{~K}$ [61] and have been shown to be Fourier transform limited below 2 K [62]. Our architecture will, therefore, be fully functional at an easily accessible temperature of $4 \mathrm{~K}$. At zero strain, they are given by the basis states $\left\{M_{1, \ldots, 6}\right\}=\left\{E_{2}, E_{1}, E_{x}, E_{y}, A_{1}, A_{2}\right\}$, neglecting a small mixture of the $E_{x, y}$ and $E_{1,2}$ states due to spin-spin interaction. The basis states $E_{x}$ and $E_{y}$ have electronic spin zero, while the others $\left(A_{1,2}\right.$ and $\left.E_{1,2}\right)$ are equal superpositions of spin $\pm 1[14,63]$. For our scheme, we apply an electric field in the $x$ direction $\left(\mathcal{E}_{x}\right)$ to lift the degeneracy of the spin-zero states in the excited-state manifold. This greatly reduces the sensitivity to rogue strain or electric field influences in the $y$ direction and, thus, makes the system more robust. $\mathcal{E}_{x}$ can be adjusted at each site to bring different $\mathrm{NV}^{-}$centers to the same resonance frequency. We choose $\left|0_{E}\right\rangle=\left|E_{x}\right\rangle+\epsilon$ and $\left.\left|1_{E}\right\rangle|=| M_{5}\right\rangle=$ $0.98\left|A_{1}\right\rangle+0.17\left|A_{2}\right\rangle+\epsilon$, where $\epsilon$ represents negligible contributions from other basis states. For this setting, we find $\delta=2 \pi \times 2.71 \mathrm{GHz}$, which is far greater than the homogeneous optical half-width of the chosen transitions, $\gamma=2 \pi \times 11 \mathrm{MHz}$. We note that, although the $\mathrm{NV}^{-}$is not a simple four-level system [Fig. 2(a)], all other allowed transitions are detuned even farther from the excitation frequency $\omega$ and can be neglected. Thus, we have the properties required for entanglement distribution based on (a)

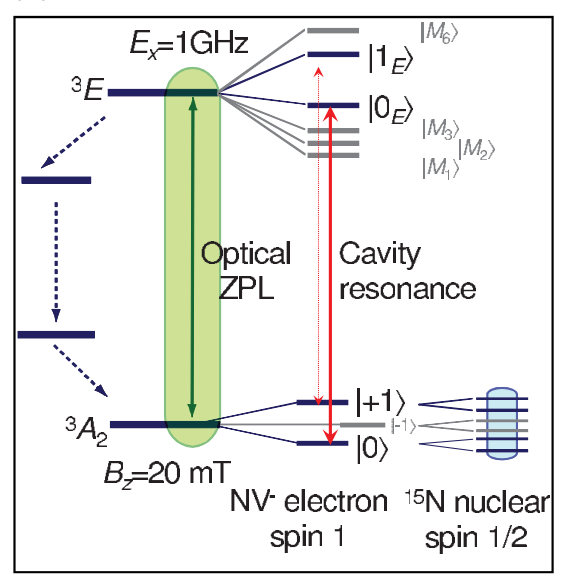

Energy Levels (b)

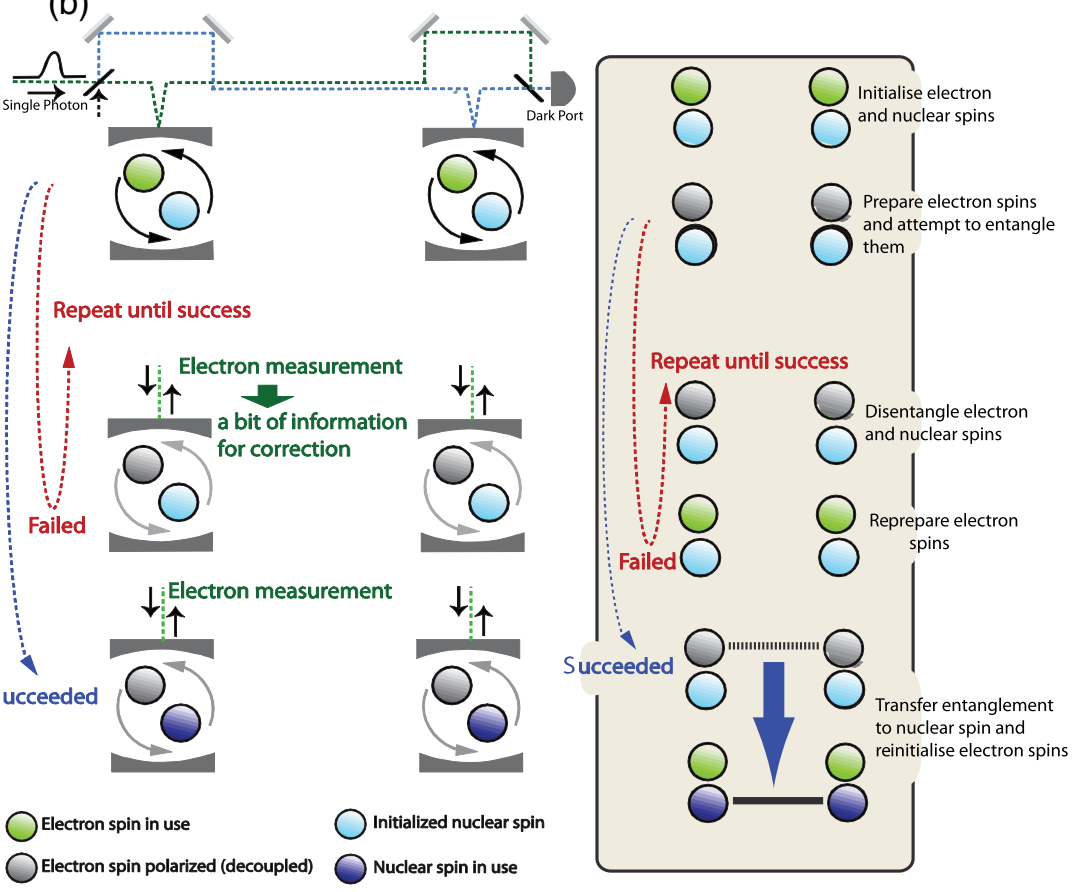

FIG. 2. $\mathrm{NV}^{-}$center is shown as a definite example of the artificial atom to realize the module. Its energy level structure for a lowtemperature, low-strain sample [14,64] is illustrated in (a). A static magnetic field of approximately $20 \mathrm{mT}$ is used to separate the $m_{s}= \pm 1$ levels. The $\mathrm{NV}^{-}$center possesses both an electron spin and a ${ }^{15} \mathrm{~N}$ nuclear spin, which will be used to store and grow a cluster state for quantum information processing. Panel (b) illustrates how the storage of entanglement in the nuclear spins is achieved. The protocol works as a repeat-until-success scheme for entangling the remote electron spins using a single photon-based Michelson interferometer. The successful event is the detection of a photon at the dark port. Once this has been achieved, the electrons are entangled with the nuclear spins followed by measurement of the electron spins to disentangle them. This allows our entanglement of the electron spins to be transferred to the nuclear spins [7]. Otherwise, the electron and nuclear spins will be decoupled completing the entangling cycle, only the electron spin reinitiated, and the protocol started again. 
state-selective reflection using the $\mathrm{NV}^{-}$center electron spin states $|0\rangle$ and $|+1\rangle$.

\section{A. Quantum nondemolition detection}

The conditional reflection of a photon from a module allows us to perform a quantum nondemolition (QND) measurement of the $\mathrm{NV}^{-}$state [52] (see Supplemental Material [58]). The measurement sequence consists of a photon measurement followed by a qubit flip and a second photon measurement. For the photon measurement, a single photon is sent to a module, and will be reflected and detected if the $\mathrm{NV}^{-}$center is in the state $|0\rangle$, and lost otherwise. The qubit flip is achieved by a microwave $\pi$ pulse. A photon detection would be expected with certainty for one of the photon measurements under ideal conditions, while the absence of a detection event would indicate leakage of the $\mathrm{NV}^{-}$center from the qubit subspace to the $|-1\rangle$ state. The destructiveness of this measurement depends on the probability of exciting the $\mathrm{NV}^{-}$center and the subsequent spin-flip probability. The measurement needs to undergo several repetitions to make up for finite photon collection efficiency, thereby increasing the spinflip probability. Nonetheless, we find that, even when taking into account all known adverse effects in the $\mathrm{NV}^{-}$center, it is possible to achieve a measurement error rate of $\epsilon_{\mathrm{QND}}=0.1 \%$ even for a finite collection efficiency of $\eta^{2}=0.8$, which is sufficient for fault-tolerant computation (see Sec. V). A working cooperativity $C=20$ is sufficient, which is realistically achievable with currently available microcavity technology $[65,66]$. The error rate can be reduced further by increasing $C$, but at much larger cooperativities the nonzero spin-flip probability for each measurement limits the QND detection. The threshold for fault-tolerant quantum computation is reached at $C=2.8$ for a detection efficiency $\eta^{2}=0.8$.

\section{B. Remote entanglement}

We begin by initializing each electron spin to $|0\rangle$ followed by rotating to $\frac{1}{\sqrt{2}}(|0\rangle+|+1\rangle)$ using a polarized driving field in a few nanoseconds. A single-photon pulse is then sent onto the interferometer [Fig. 2(b)] and the dark port monitored. We repeat this procedure until the entanglement is heralded by the successful detection of a photon at the dark port. In such a situation, we have the electron spins in the entangled state $\frac{1}{\sqrt{2}}[|0\rangle|+1\rangle-|+1\rangle|0\rangle]$ (see Supplemental Material for details [58]). This is made possible by the good cycling properties of the $\mathrm{NV}^{-}$ transition $|0\rangle \rightarrow\left|E_{x}\right\rangle$ [14]. We note, furthermore, that the deionization process $\mathrm{NV}^{-} \rightarrow \mathrm{NV}^{0}$, and the resulting dynamical spectral diffusion, is rendered impossible by using only one single-photon excitation in the interferometer at a time [6].

In the $\mathrm{NV}^{-}$implementation of our module, the nuclear spin is a long-lived quantum memory that will, in our architecture, be designated to store one node of a cluster state [67]. Our scheme creates entanglement between the electrons of the two $\mathrm{NV}^{-}$centers $[7,8,44,46]$. The transfer of the entanglement to the nuclear spin memories [9] is done through the Ising component of the hyperfine coupling $\left(A_{\|} \sim 3.03 \mathrm{MHz}[68]\right)$, which is tuned by the external magnetic field of $B \sim 20 \mathrm{mT}$ to give a conditional phase on the state of the two spins. The amount of entanglement oscillates in time from zero to maximum. At time $\tau$, setting $\pi$ points of the oscillation, the effective gate becomes a controlled-phase gate, while at the $2 \pi$ point it gives identity. The hyperfine coupling is always present but is effectively turned off while the electron spin is in the polarized state $|0\rangle[9]$.

Putting this together, the complete nuclear spin entanglement protocol begins with both electron spins and both nuclear spins polarized in their ground states (achieved via the quantum nondemolition measurement). The electron spin is then rapidly rotated to the $|+1\rangle$ state via a $\pi / 2 Y$ rotation, and a rf pulse of frequency $\nu=A_{\|}-B g_{n} \mu_{n}+$ $A_{\perp}^{2} /\left(2 D+2 B g_{e} \mu_{e}\right) \sim 3 \mathrm{MHz}$ is applied to rotate the nuclear spin by $\pi / 4$. The electron spin is then rotated back to its ground state $|0\rangle$. This has prepared the nuclear spin into the $\left|n_{+}\right\rangle$state. We then rotate the electron back into the $|+\rangle$ state to attempt an electron-electron bond via the optical transitions. The hyperfine coupling turns on when the photonic entangling protocol is initiated by the electron spin rotation, but a spin-echo-like sequence can be used to disentangle the electron and nuclear spins at any time we require. If the gate has succeeded, we perform a $\pi / 4 Y$ rotation on one of two electron spins and wait until the hyperfine interaction maximally entangles the electron and nuclear spins within each node. A $\pi / 4 Y$ rotation is then performed on the electron spin of each module followed by its measurement in the computational basis. This completes the transfer of the entangled link to the nuclear spins. If the entanglement distribution has failed, the protocol will be repeated until a success is heralded, as illustrated in Fig. 2(b). We note that it is not necessary to reinitialize the nuclear spin prior to each attempt as our next attempt begins at the $2 \pi$ point of the hyperfine interaction where the electron and nuclear spins are decoupled (further information is given in the Appendix and the Supplemental Material [58]). This is one of the key elements to extend the generation of the Bell state to general cluster states.

\section{SHARING ENTANGLED STATES BETWEEN THREE MODULES}

The next step is to extend our cluster of two nuclear spin qubits to three (by adding one). We begin with an entangled pair stored in the nuclear spins of modules A and B as shown in Fig. 3. A new entanglement bond on the electron spins in modules $\mathrm{B}$ and $\mathrm{C}$ is created using the same repeatuntil-success protocol, though only the nuclear spin in $\mathrm{C}$ will be initialized. Once the entanglement between the 
electronic qubits is created, the entanglement will be transferred to the nuclear spins using the hyperfine coupling described previously.

However, this time, the nuclear spin in module B is in use, carrying information established at a previous time in the protocol. In general, for the protocol to be useful, it must allow us to preserve with high fidelity the existing entanglement stored in the nuclear spins (in this case, A and $\mathrm{B}$ ), while using the electron spins to create entanglement with additional modules (C). However, due to the permanent hyperfine coupling, photon loss occurring at random times may induce decoherence in the states stored in the nuclear spins. By introducing a time-sequenced entangling procedure, we can suppress this decoherence. Furthermore, by using spin-echo-like sequences to decouple the electron spins from their surrounding environment, we may extend their coherence time. The clock for the hyperfine coupling sequence starts when the photonic entangling protocol is initiated (that is, when the electron spin is rotated out of a polarized $|0\rangle$ state). If the entangling protocol fails, the system waits until the spin-echo sequence decouples the electron and nuclear spins. At this point, the nuclear system recovers coherence and the information stored on the nuclear spin remains untouched until the protocol succeeds. This process is illustrated in Fig. 3. Once the new entangling bond is established, indicated by a heralding signal, we again wait until the spin echo decouples the electron and nuclear spins. We then perform a single $\pi / 4 Y$ rotation on one of the two electron spins and wait until the hyperfine interaction maximally entangles the electron and nuclear spins within each the nodes. An $X$-basis measurement is performed on each electron (via a $\pi / 4 Y$ rotation and computational basis readout) to transfer the new bond to the nuclear system.

\section{A. Cluster states}

Repeating this with additional modules, we can generate an arbitrary cluster state. To illustrate this, we depict in

(a)

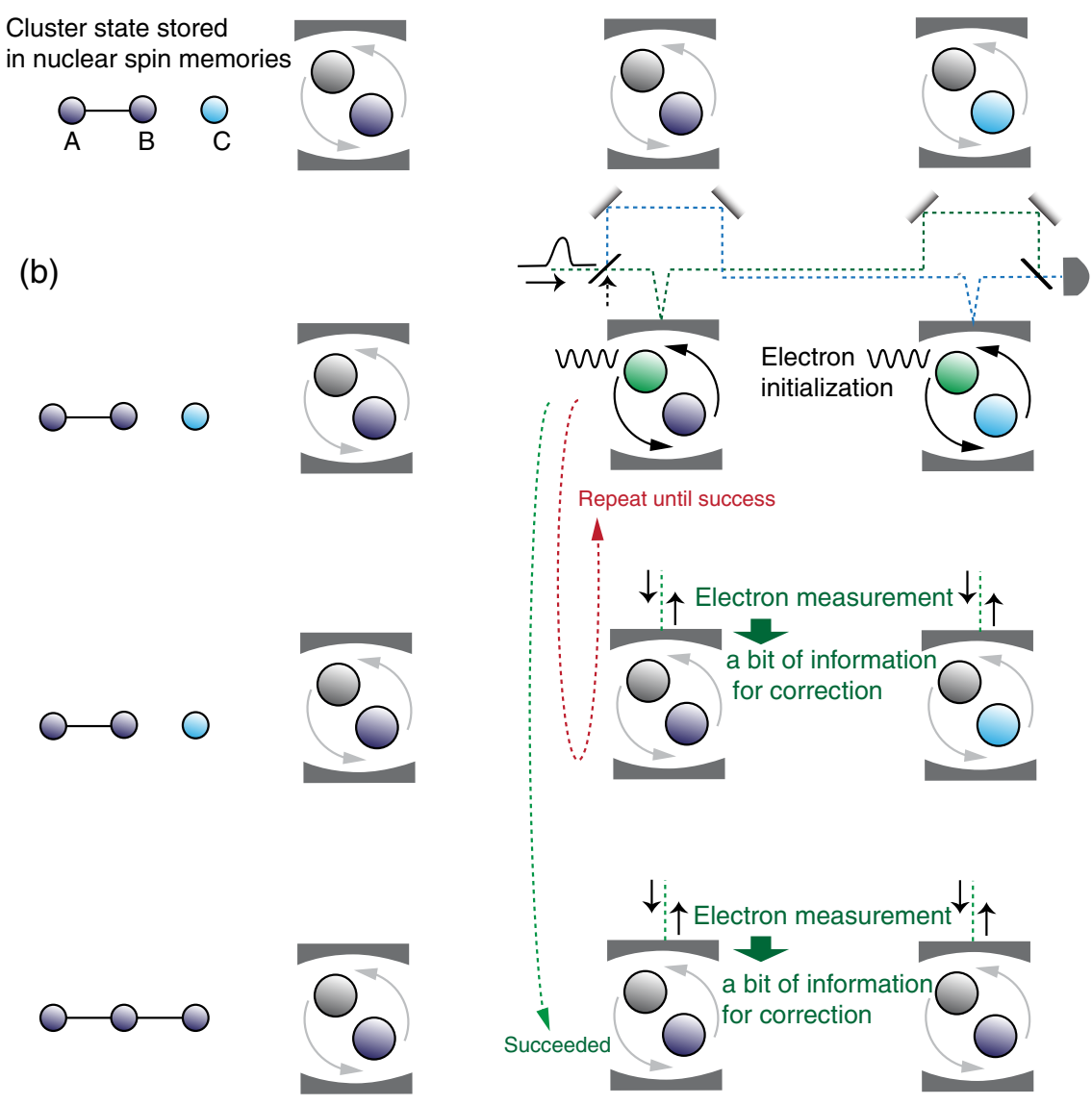

FIG. 3. The repeat-until-success protocol is accurately time sequenced. This is required by the nature of the coupling, as entanglement between the electron spin and nuclear spin oscillates. Upon failure, we wait until the $2 \pi$ point in the entangling cycle, where the nucleus and electron are decoupled. The nuclear spin is consequently protected from feedback errors through the hyperfine coupling by accurately timing the reinitialization of the electron spins. When the distribution of entanglement between two electrons succeeds, the entanglement bond will be transferred to the nuclear spins by waiting until a $\pi$ point where the electron and nuclear spins are maximally entangled. 


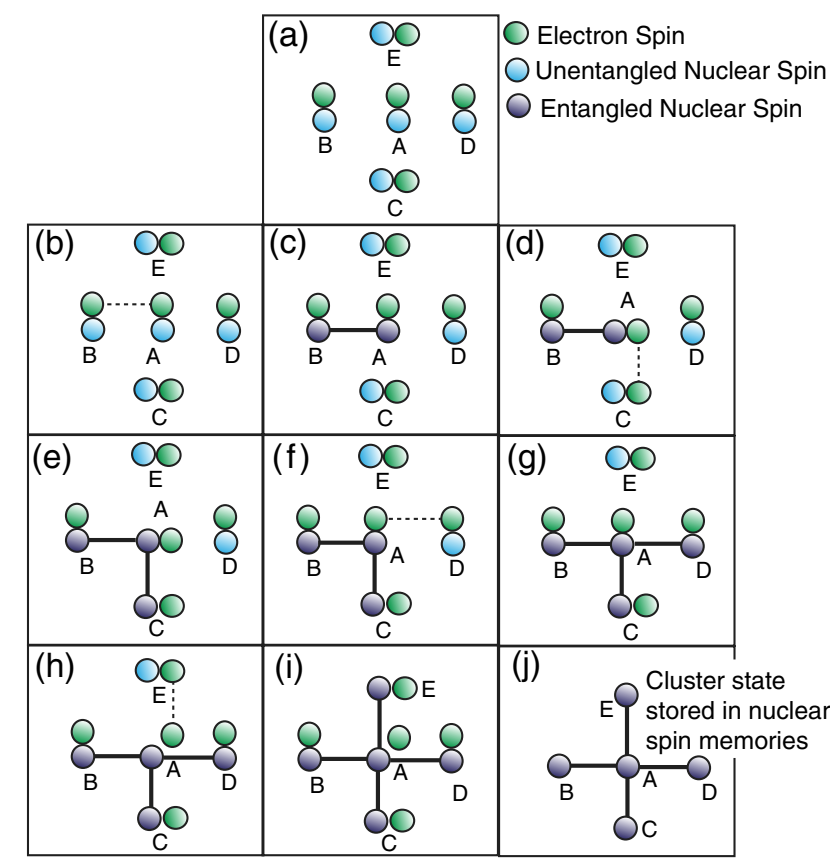

...... Electron Spin Entangled Link — Nuclear Spin Entangled Link
Fig. 4 the sequence of operations for creating a cross cluster composed of five nuclear spins.

We are particularly interested in generating the three-dimensional topological cluster state [illustrated in Fig. 5(a)] capable of supporting fault-tolerant quantum computation $[33,34]$. Topological models of error correction $[69,70]$ exhibit relatively high tolerance to errors and are particularly well suited to architectures due to their simple underlying structure $[12,39,40,71,72]$. The topological cluster state is particularly useful in the context of our repeat-until-success protocol, as it is inherently robust against missing bonds, which will be heralded. These missing bonds can be processed in the classical interpretation of measurement results, without any modification to the quantum circuit [35]. To prepare the topological cluster state, each physical qubit is entangled with its four nearest neighbors; hence, a dagger-shaped cluster state is the fundamental unit, independent of the size of the network, highlighted by the blue bond in Fig. 5(b). Four entangling steps are required to create this fundamental state with five modules.

\section{BENCHMARKING THE PHOTONIC ARCHITECTURE}

To process quantum information with a threedimensional topological cluster state, the state is consumed

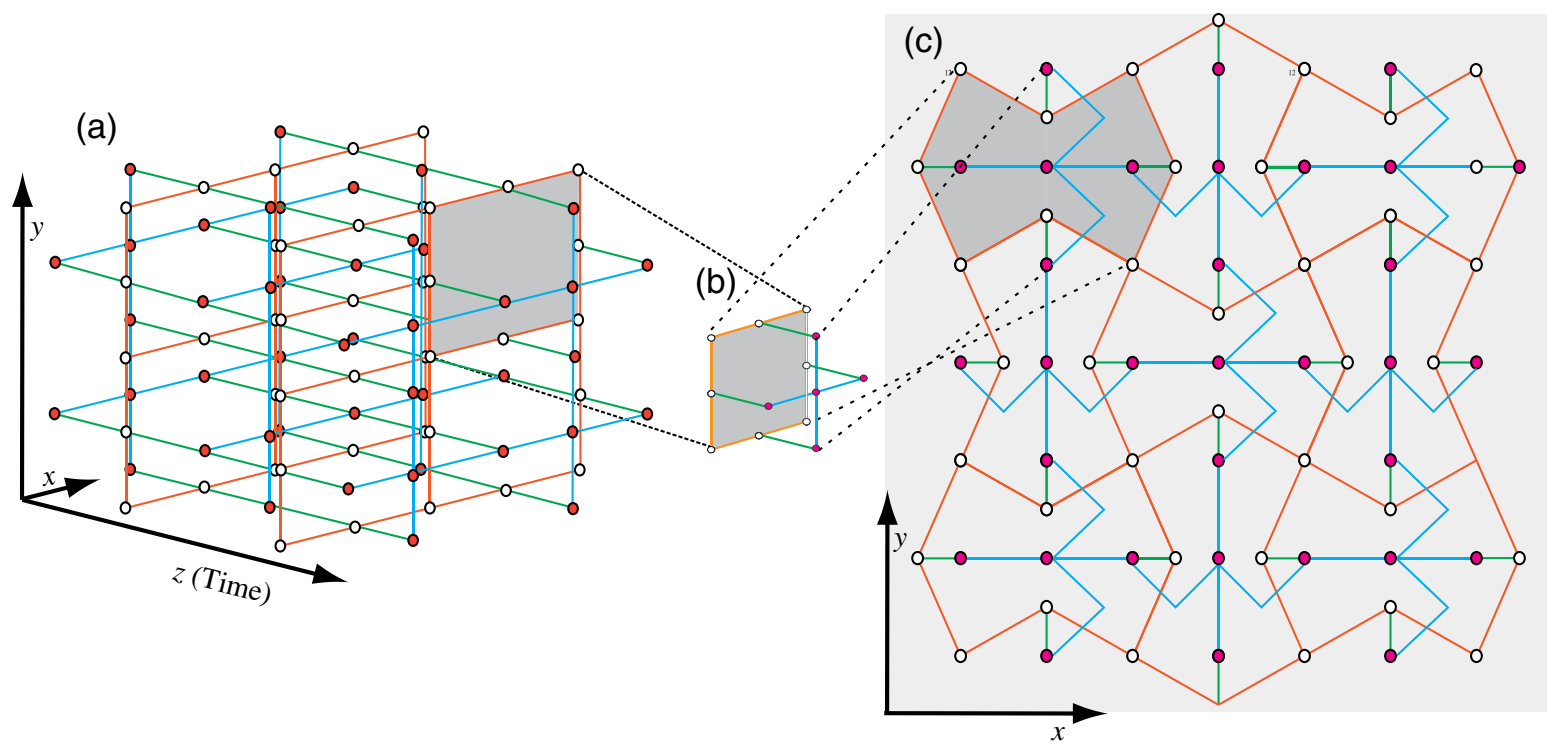

FIG. 5. Three-dimensional topological cluster state and module connectivity in a two-dimensional plane. (a) The topological cluster state cluster is a resource for fault-tolerant quantum computation. However, the whole state is not required at all times during the computation. Instead, only two layers of the cluster state need to be prepared and stored at any given time. (b) The physical unit cell composed of two layers. The back layer contains eight connected qubits arranged in a square (orange), while the front layer has five qubits arranged in a cross (blue). The two layers are connected by controlled-phase gates (green). Measurement of the front layer of the cluster will teleport the current state of the computer to the back layer, at which point the physical qubits we just measured can be reconnected in accordance with the geometry of the cluster state, and the information can be teleported back again. In this way, the two physical layers execute the even and odd temporal steps of the computation, allowing an arbitrarily deep computation to be performed with a fixed number of physical qubits. (c) A compact layout of modules on a two-dimensional plane. 
by measurements on physical qubits in sequential twodimensional layers, where one axis is defined as the temporal axis. These measurements create and manipulate encoded qubits defined by defects $[33,73]$. As the computation proceeds by measuring one layer at a time, the whole topological cluster state is not required to be constructed initially. Only two successive layers need to be prepared and stored at any given time, allowing us to concentrate on only two physical layers of modules. The current state of the computer is teleported back and forth between these two layers, which are refreshed and recycled to generate the entire topological cluster. Taking the center of each cell [in Fig. 5(b)], we initiate a sequence of gates to generate the dagger-shaped cluster state throughout the lattice, which generates one layer of the topological cluster state (see Supplemental Material [58]). The two layers of the module network are flattened to a two-dimensional plane, as shown in Fig. 5(c). This pattern repeats to an arbitrarily large cross section.

At a circuit level, we are interested in the threshold error rate, below which the architecture becomes fault tolerant $[33,74]$. The projective measurement of the nucleus (via the electron-nucleus hyperfine interaction) allows us to combine measurement and reinitialization of the nuclear qubit in a single step. Therefore, the depth of the quantum circuit to prepare the topological cluster state is reduced from six steps to five. We find that this reduction increases the error threshold to $0.73 \%$ [see Fig. 6(a)]. Given this threshold, our target error rate for the five relevant gates is $\sim 0.1 \%$, as this is sufficiently far below the threshold to allow significant suppression of errors using a practical number of modules [73].

The target error rate does not tell us much until it is decomposed into each physical component. Each gate consists of several physical steps and involves several sources of errors. In our case, these sources are parametrized by the nuclear and electron spin decoherence times, electron measurement efficiency, electron rotation efficiency, and timing error. As described, the sequence to generate an entangling bond is probabilistic, and the protocol repeats until success. Given that we require bonds to succeed with probability $P=99.9 \%$, if the success probably of a single attempt is $p_{c}=\eta^{2} P_{R} / 8$, the number of attempts we require is $s=\log (1-P) / \log \left(1-p_{c}\right)$. For $p_{c}=0.0625, s=107$. We consider the error rate for each gate to be the worst-case scenario, as heralded failure can be significantly higher than the error rate for unheralded errors [35].

The required fidelity for each physical parameter is shown in Fig. 6(b). Each curve is plotted assuming it is the only nonzero error, except for possible errors arising from the absorption of photons by the $\mathrm{NV}^{-}$node (see Supplemental Material [58]). The green region in Fig. 6(b) is the target for each parameter for an operational computer (though parameters in the yellow region still lead to gates (a)

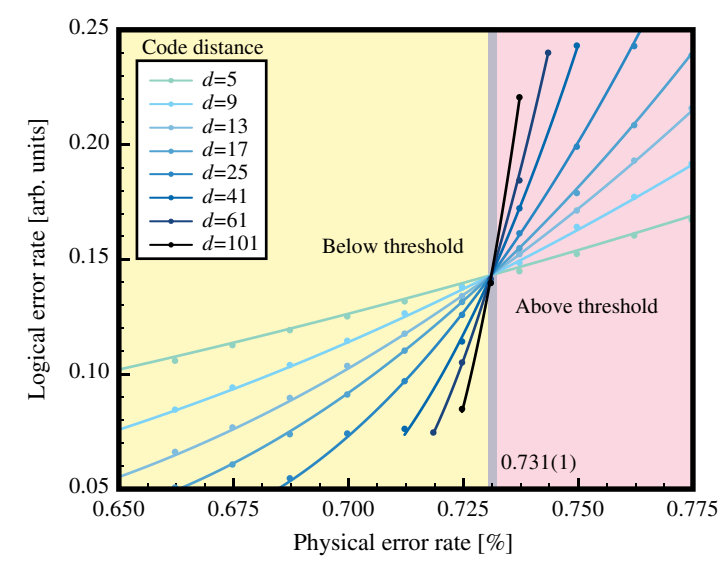

(b)

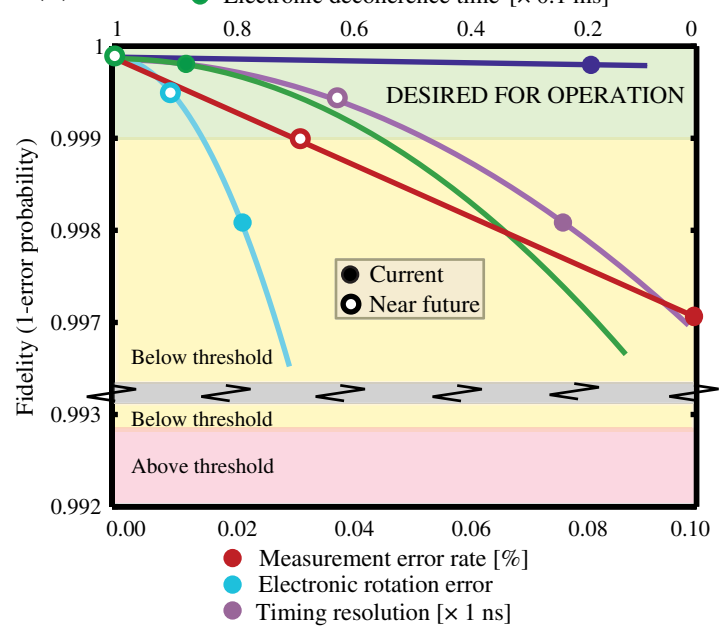

FIG. 6. Fault-tolerant thresholds and required component error rates. (a) Numerical simulation of topological error correction [74]. The logical error rate is plotted as a function of the physical error rate for various code sizes (distances $d$ ), where we assume that all gates and measurements are operating at the same error rate. Each point corresponds to at least $10^{4}$ trials. The value of the physical error rate at the intersection gives the threshold (in this case, approximately $7.3 \times 10^{-3}$ ). For physical error rates below this threshold, the logical error rate can be reduced arbitrarily by increasing the code distance. (b) The required fidelity (1-error probability) for each physical parameter. In the Supplemental Material [58] details of the error calculations are shown for various physical parameters. The dots on the lines show the current best accuracy reported, all of which already meet the required accuracy, 99.27\%. For a realistic implementation, the gate fidelity should be above $99.9 \%$, corresponding to the green region in the plot. Electronic and nuclear spin coherence times are already in this regime, and the remaining parameters may soon meet the desired accuracy given the rapid development of quantum control of such systems. The fidelity does not converge to unity due to imperfections in the $\mathrm{NV}^{-}$center. 
below the threshold). For the architecture to be fault tolerant, these errors need to be combined (see Supplemental Material [58]). Electron and nuclear decoherence is already sufficiently low [13,75-77], while the other parameters still need improvement. However, it is important to note that the required improvements are less than 1 order of magnitude, and are not limited by any currently known fundamental limitations of the $\mathrm{NV}^{-}$ system itself.

Assuming that the threshold condition is met, performance is mostly dependent on the computational cycle time, which is limited by the time taken to establish all of the electron-electron connections. For bond connections with $P=99.9 \%$, the total time required to create a nuclearnuclear bond is $3.5 \mu \mathrm{s}$, assuming $p_{c}=6.25 \%$. This time could be reduced by lowering the required connection efficiency and exploiting the robustness of the topological code to missing bonds [35]. The quantum circuit takes five steps to construct each cross-sectional layer of the topological cluster state. Hence, a unit cell of the cluster is prepared every $\sim 30 \mu \mathrm{s}$. To implement an algorithm on the computer, we create pairs of defects in the cluster. The volume of cluster allocated to pairs of defects represents the degree of error correction, parametrized by the distance between defects $d$. For a logical error rate $p_{L} \leq 10^{-18}$, $d \geq 32$ is required [73]. Therefore, a logical cell requires $V=\left(\frac{5 d}{4}\right)^{3}=40^{3}$ cluster cells. To perform a logical CNOT gate requires a cluster volume $2 \times 2$ in cross section and two logical cells in temporal depth. Hence, it takes $3.4 \mathrm{~ms}$ for $p_{c}=6.25 \%$ (a clock frequency of $\sim 295 \mathrm{~Hz}$ ). This rate can be further improved by better optical efficiencies, but is ultimately limited by the hyperfine interaction of the $\mathrm{NV}^{-}$ node used for nuclear spin operations. If we assume a deterministic electron-electron connection, a logical CNOT gate would take approximately $960 \mu \mathrm{s}(\sim 1 \mathrm{kHz})$ as the system becomes rate limited by nuclear measurement (see Supplemental Material [58]).

\section{CONCLUSION}

As we have seen, a simple module can form the basis of a scalable quantum computer architecture. The architecture is naturally distributed, and hence is applicable to quantum communication [78]. Such a network may be local or global, with local networks connected by quantum communication channels. In this case, the distance between the modules may become orders of magnitude larger. The time delay due to the communication distance may be mitigated by the longlived memory inside the module. With increased distance between modules, photon loss would increase, reducing the success probability of the entangling protocol. However, long-distance communication does not necessarily require $P=99.9 \%$. Instead, with $P=99.0 \%$, the number of attempts can be reduced to $s=71$ for $p_{c}=6.25 \%$.

We find that physical requirements of our architecture are broadly consistent with present technology. However, while technological developments might further help to meet these requirements, physical requirements may be found to be less stringent with a more sophisticated adaptive error analysis.

\section{ACKNOWLEGMENTS}

We thank Austin Fowler and Andrew Greentree for valuable discussions. We acknowledge partial support from FIRST, NTT, and NICT in Japan, the Austrian Science Fund (FWF) through the Wittgenstein Prize, the FFG through the project PLATON, and the EU through the project DIAMANT. K. B. and T. N. acknowledge support from the FWF Doctoral Programme CoQuS (W1210).

\section{APPENDIX: DIAMOND MODULE OPERATIONS}

The key to our architecture is the physical system implementing the module. How well the $\mathrm{NV}^{-}$center could faithfully realize the module dictates the requirements for physical parameters.

\section{A. Description of the $\mathrm{NV}^{-}$center}

The dynamics of the $\mathrm{NV}^{-}$center, consisting of the electron spin-1 ${ }^{3} A_{2}$ manifold and the nuclear spin- $1 / 2$ system, can be described by the Hamiltonian $H=H_{e}+H_{n}+H_{e-n}$. The electron spin's ground-state Hamiltonian is given by $[79,80]$

$$
H_{e}=\hbar\left(D S_{z}^{2}+E\left[S_{x}^{2}-S_{y}^{2}\right]+g_{e} \mu_{B} B S_{z}\right),
$$

which represents a zero-field splitting $(D / 2 \pi=2.87 \mathrm{GHz})$, a strain-induced splitting (typically $E / 2 \pi \sim 1-10 \mathrm{MHz}$ ), and a magnetic field-induced splitting $\left(g_{e} \mu_{B} B\right)$, where $\mu_{B}$ is the Bohr magneton and $g_{e}=2.0$ is the $g$ factor. In this Hamiltonian, $S_{z}, S_{x}, S_{y}$ are the usual spin-1 operators. With an externally applied magnetic field $B \sim 20 \mathrm{mT}$, our electron spin qubit levels $|0\rangle$ and $|+1\rangle$ are far detuned from the $|-1\rangle$ energy level, supporting our electron spin qubit. The effects of the strain-induced splitting present in CVD diamond are made negligible by the large applied magnetic field. The nuclear spin Hamiltonian $H_{n}=$ $-\hbar g_{n} \mu_{n} B I_{z}$ represents a magnetic field-induced splitting of the ${ }^{15} \mathrm{~N}$ nuclear spin, where $\mu_{n}$ is the nuclear magneton and $g_{n}=-0.566$ is the nuclear $g$ factor. $I_{z}$ is the usual Pauli $Z$ spin-1/2 operator.

The hyperfine coupling between the electron and the nuclear spins is given by [68]

$$
H_{e-n}=\hbar A_{\|} S_{z} I_{z}+\frac{\hbar A_{\perp}}{2}\left(S_{+} I_{-}+S_{-} I_{+}\right),
$$

where $S_{ \pm}\left(I_{ \pm}\right)$are the electron spin (nuclear spin) raising and lowering operators, respectively. This coupling includes an Ising part with coupling strength $A_{\|} / 2 \pi \sim$ $3.03 \mathrm{MHz}$ and an exchange part with coupling constant 
$A_{\perp} / 2 \pi \sim 3.65 \mathrm{MHz}$ [68]. With $B \sim 20 \mathrm{mT}$, the exchange coupling is far off resonance, resulting only in a small dispersive phase shift. This results in a natural controlledphase gate that operates on a time scale $\tau \sim \pi /\left[A_{\|}+\frac{A_{\perp}^{2}}{2 \lambda}\right] \sim$ $165 \mathrm{~ns}$, where $\lambda$ is the difference in the Larmor frequencies between the electron and nuclear spin levels.

An external microwave or $\mathrm{rf}$ driving of amplitude $\Omega_{0}$ is used to perform the electron and nuclear spin rotations. The driving Hamiltonian can be expressed as

$$
H_{d}=\hbar \Omega_{0} \cos \left(\omega_{d} t+\phi\right)\left(S_{x}-\frac{g_{n} \mu_{n}}{g_{e} \mu_{B}} I_{x}\right),
$$

where the frequency $\omega_{d}$ is chosen appropriately to determine whether we drive the electron or nuclear spin, with $\phi$ representing an initial phase offset. By using a polarized field, electron spin rotations can be achieved with high fidelity in at most a few nanoseconds. The nuclear spin operations are much slower due to the weak gyromagnetic ratio but can be achieved (with high fidelity) in a few microseconds by using the hyperfine coupling to enhance the natural nuclear spin splitting.

\section{B. Entangling nuclear spins in more detail}

We consider, in a little more detail, the establishment of entanglement between the two nuclear spins. We assume the overall system is initialized in their restrictive ground states (both electron and nuclear spins). The electron spins are rotated to the $|+1\rangle$ state and a $\pi$ rotation performed on the nuclear spins to prepare them in the $\left|n_{+}\right\rangle$state. The electron spins can then be rotated back to the $|0\rangle$ state where they are effectively decoupled from the nuclear spins. To begin the entanglement protocol, we now rotate the electron spins to the $|+\rangle$ state and interact them with the photon entering the Michelson interferometer. Two possible events occur. Either a photon is detected at the dark port or it is not. Let us deal with this second event first.

\section{Unsuccessful detection at the dark port}

In the situation where the photon has not been detected at the dark port, it has either gone out the bright port or been loss somewhere in the circuit. Given that we do not know which of these two detreminal events occurred, each of our electron spins is left in an indeterminate state (say, $\rho=$ $\left.\frac{1}{2}[|0\rangle\langle 0|+|+1\rangle\langle+1|]\right)$. Let us focus on only one of the electron and nuclear spins now. The always-on hyperfine controlled Z (CZ) coupling means the electron and nuclear spins are attempting to interact with one another. In such a case, we can show that the electron and nuclear spin evolve as

$$
\begin{aligned}
& \frac{1}{2}[|0\rangle\langle 0|+|+1\rangle\langle+1|] \otimes\left|n_{+}\right\rangle\left\langle n_{+}\right| \rightarrow \\
& \quad \frac{1}{2}|0\rangle\left\langle 0|\otimes| n_{+}\right\rangle\left\langle n_{+}\left|+\frac{1}{2}\right|+1\right\rangle\left\langle+1|\otimes| n_{\theta}\right\rangle\left\langle n_{\theta}\right|,
\end{aligned}
$$

where $\left|n_{\theta}\right\rangle=\frac{1}{\sqrt{2}}\left[\left|n_{0}\right\rangle+e^{i A_{\|} t}\left|n_{1}\right\rangle\right]$, with $A_{\|}$the parallel component of the hyperfine interaction and $t$ the time since the electron and nuclear spin state entangling. Thus, it seems that the decoherence in our electron spin has caused us to lose coherence in the nuclear spin. However at $A_{\|} t=$ $2 \pi$ point, the electron and nuclear spins naturally decouple (the same effect can be achieved using a spin-echo sequence on the electron spin). When they are decoupled, we reinitialize the electron spin in the $\left|e_{+}\right\rangle$to restart the entangling protocol again, or in the $|0\rangle$ state for the next attempt whenever required.

\section{Successful detection at the dark port}

Upon a successful detection event (and also assuming a spin-echo sequence has been used to keep the electron and nuclear spins decoupled), our combined electron-nuclear spin system can be represented as

$$
\frac{1}{\sqrt{2}}[|0\rangle|+1\rangle-|+1\rangle|0\rangle]\left|n_{+}\right\rangle\left|n_{+}\right\rangle .
$$

We then perform a $\pi / 4$ rotation on the first electron spin and wait for the CZ-based hyperfine interaction to maximally entangle the electron and nuclear spins (this occurs at $A_{\|} t=\pi$ ). This results in the state

$$
\frac{1}{2}(|+10-\rangle+|-11-\rangle-|-00+\rangle-|+01+\rangle),
$$

using the notation convention $\left|e_{1}, e_{2}, n_{1}, n_{2}\right\rangle$. Finally, a $\pi / 4$ $Y$ rotation is performed on the electron spins of each module before their measurement in the computational basis. This results in the combined nuclear spin state (up to Pauli corrections that are classically tracked)

$$
\frac{1}{2}\left[\left|n_{0}\right\rangle\left|n_{-}\right\rangle+\left|n_{1}\right\rangle\left|n_{+}\right\rangle\right],
$$

that is, a two qubit nuclear spin cluster.

\section{Coherence properties}

It is critical to mention the coherence properties of our electron-nuclear spin as this can vary significantly. Here, we assume that a single ${ }^{15} \mathrm{NV}^{-}$center is created on isotopically pure $(99.9+12 \mathrm{C})$ diamond substrate [75] and that our module will operate at low temperature (4-20 K) rather than at room temperature. In such a case, it has been reported that $T_{1}$ of the electron spin is greater than $1 \mathrm{~s}$, while $T_{2}^{*} \sim 90 \mu \mathrm{s}[76,77]$ with $T_{2}$ much longer [75]. The nuclear spin $T_{1}$ and $T_{2}$ are at least $0.2 \mathrm{~s}$ at present [13]. The limiting coherence parameter in this design is the $T_{2}^{*}$ of the electron spin during the 165 ns controlled-phase gate. However, with Gaussian decay having the form $\exp \left[-\left(2 t / T_{2}^{*}\right)^{2}\right]$, the error associated with this is small in principle $\left(<10^{-5}\right)[75]$. 
[1] T. D. Ladd, F. Jelezko, R. Laflamme, Y. Nakamura, C. Monroe, and J. L. O'Brien, Quantum Computers, Nature (London) 464, 45 (2010).

[2] S. J. Devitt, W. J. Munro, and K. Nemoto, Quantum Error Correction for Beginners, Rep. Prog. Phys. 76, 076001 (2013).

[3] G. Davies and M. F. Hamer, Optical Studies of the $1.945 \mathrm{eV}$ Vibronic Band in Diamond, Proc. R. Soc. A 348, 285 (1976).

[4] R. T. Harley, M. J. Henderson, and R. M. Macfarlane, Persistent Spectral Hole Burning of Colour Centres in Diamond, J. Phys. C 17, L233 (1984).

[5] E. Van Oort, N. B. Manson, and M. Glasbeek, Optically Detected Spin Coherence of the Diamond NV Centre in its Triplet Ground State, J. Phys. C 21, 4385 (1988).

[6] M. W. Doherty, N. B. Manson, P. Delaney, F. Jelezko, J. Wrachtrup, and L.C. Hollenberg, The Nitrogen-Vacancy Colour Centre in Diamond, Phys. Rep. 528, 1 (2013).

[7] L. Childress, J. M. Taylor, A. S. Srensen, and M. D. Lukin, Fault-Tolerant Quantum Repeaters with Minimal Physical Resources and Implementations Based on Single-Photon Emitters, Phys. Rev. A 72, 052330 (2005).

[8] L. Childress, J. M. Taylor, A. S. Sørensen, and M. D. Lukin, Fault-Tolerant Quantum Communication Based on SolidState Photon Emitters, Phys. Rev. Lett. 96, 070504 (2006).

[9] M. V. G. Dutt, L. Childress, L. Jiang, E. Togan, J. Maze, F. Jelezko, A. S. Zibrov, P. R. Hemmer, and M. D. Lukin, Quantum Register Based on Individual Electronic and Nuclear Spin Qubits in Diamond, Science 316, 1312 (2007).

[10] S. C. Benjamin, D. E. Browne, J. Fitzsimons, and J. J. L. Morton, Brokered Graph-State Quantum Computation, New J. Phys. 8, 141 (2006).

[11] L. Jiang, J. M. Taylor, A. S. Sørensen, and M. D. Lukin, Distributed Quantum Computation Based on Small Quantum Registers, Phys. Rev. A 76, 062323 (2007).

[12] N. Yao, L. Jiang, A. Gorshkov, P. Maurer, G. Giedke, J. Cirac, and M. Lukin, Scalable Architecture for a Room Temperature Solid-State QuantumInformation Processor, Nat. Commun. 3, 800 (2012).

[13] P. C. Maurer, G. Kucsko, C. Latta, L. Jiang, N. Y. Yao, S. D. Bennett, F. Pastawsk, D. Hunger, N. Chisholm, M. Markham, D. J. Twitchen, J. I. Cirac, and M. D. Lukin, Room-Temperature Quantum Bit Memory Exceeding One Second, Science 336, 1283 (2012).

[14] E. Togan, Y. Chu, A. S. Trifonov, L. Jiang, J. Maze, L. Childress, M. V. G. Dutt, A. S. Sørensen, P. R. Hemmer, A.S. Zibrov, and M. D. Lukin, Quantum Entanglement between an Optical Photon and a Solid-State Spin Qubit, Nature (London) 466, 730 (2010).

[15] F. Jelezko, T. Gaebel, I. Popa, A. Gruber, and J. Wrachtrup, Observation of Coherent Oscillations in a Single Electron Spin, Phys. Rev. Lett. 92, 076401 (2004).

[16] P. Neumann, N. Mizuochi, F. Rempp, P. Hemmer, H. Watanabe, S. Yamasaki, V. Jacques, T. Gaebel, F. Jelezko, and J. Wrachtrup, Multipartite Entanglement among Single Spins in Diamond, Science 320, 1326 (2008).

[17] R. Hanson, V. V. Dobrovitski, A. E. Feiguin, O. Gywat, and D. D. Awschalom, Coherent Dynamics of a Single Spin Interacting with an Adjustable Spin Bath, Science 320, 352 (2008).
[18] L. Jiang, J. S. Hodges, J. R. Maze, P. Maurer, J. M. Taylor, D. G. Cory, P. R. Hemmer, R. L. Walsworth, A. Yacoby, A. S. Zibrov, and M. D. Lukin, Repetitive Readout of a Single Electronic Spin via Quantum Logic with Nuclear Spin Ancillae, Science 326, 267 (2009).

[19] P. Neumann, J. Beck, M. Steine, F. Rempp, H. Fedder, P. R. Hemmer. J. Wrachtrup, and F. Jelezko, Single-Shot Readout of a Single Nuclear Spin, Science 329, 542 (2010).

[20] P. Neumann, R. Kolesov, B. Naydenov, J. Beck, F. Rempp, M. Steiner, V. Jacques, G. Balasubramanian, M. L. Markham, D. J. Twitchen, S. Pezzagna, J. Meijer, J. Twamley, F. Jelezko, and J. Wrachtrup, Quantum Register Based on Coupled Electron Spins in a Room-Temperature Solid, Nat. Phys. 6, 249 (2010).

[21] B. B. Buckley, G. D. Fuchs, L. C. Bassett, and D. D. Awschalom, Spin-Light Coherence for Single-Spin Measurement and Control in Diamond, Science 330, 1212 (2010).

[22] L. Robledo, L. Childress, H. Bernien, B. Hensen, P. F. A. Alkemade, and R. Hanson, High-Fidelity Projective Read-out of a Solid-State Spin Quantum Register, Nature (London) 477, 574 (2011).

[23] T. van der Sar, Z. H. Wang, M. S. Blok, H. Bernien, T. H. Taminiau, D. M. Toyli, D. A. Lidar, D. D. Awschalom, R. Hanson, and V. V. Dobrovitski, Decoherence-Protected Quantum Gates for a Hybrid Solid-State Spin Register, Nature (London) 484, 82 (2012).

[24] F. Dolde, I. Jakobi, B. Naydenov, N. Zhao, S. Pezzagna, C. Trautmann, J. Meijer, P. Neumann, F. Jelezko, and J. Wrachtrup, Room-Temperature Entanglement between Single Defect Spins in Diamond, Nat. Phys. 9, 139 (2013).

[25] H. Bernien, B. Hensen, W. Pfaff, G. Koolstra, M. S. Blok, L. Robledo, T. H. Taminiau, M. Markham, D. J. Twitchen, L. Childress and R. Hanson, Heralded Entanglement between Solid-State Qubits Separated by Three Metres, Nature (London) 497, 86 (2013).

[26] Y.-S. Park, A. K. Cook, and H. Wang, Cavity QED with Diamond Nanocrystals and Silica Microspheres, Nano Lett. 6, 2075 (2006).

[27] D. Englund, B. J. Shields, K. Rivoire, F. Hatami, J. Vuckovic, H. Park, and M. D. Lukin, Deterministic Coupling of a Single Nitrogen Vacancy Center to a Photonic Crystal Cavity, Nano Lett. 10, 3922 (2010).

[28] A. Faraon, P. E. Barclay, C. Santori, K.-M. C. Fu, and R. G. Beausoleil, Resonant Enhancement of the Zero-Phonon Emission from a Colour Centre in a Diamond Cavity, Nat. Photonics 5, 301 (2011).

[29] D. Englund, A. Faraon, I. Fushman, N. Stoltz, J. Vuckovic, and P. Petroff, Controlling Cavity Reflectivity with a Single Quantum Dot, Nature (London) 450, 857 (2007).

[30] E. Knill, Quantum Computing with Realistically Noisy Devices, Nature (London) 434, 39 (2005).

[31] D. Bacon, Operator Quantum Error Correcting Subsystems for Self-Correcting Quantum Memories, Phys. Rev. A 73, 012340 (2006).

[32] R. Raussendorf and J. Harrington, Fault-Tolerant Quantum Computation with High Threshold in Two Dimensions, Phys. Rev. Lett. 98, 190504 (2007). 
[33] R. Raussendorf, J. Harrington, and K. Goyal, Topological Fault-Tolerance in Cluster State Quantum Computation, New J. Phys. 9, 199 (2007).

[34] A. Fowler and K. Goyal, Topological Cluster State Quantum Computing, Quantum Inf. Comput. 9, 721 (2009).

[35] S. D. Barrett and T. M. Stace, Fault Tolerant Quantum Computation with Very High Threshold for Loss Errors, Phys. Rev. Lett. 105, 200502 (2010).

[36] A. G. Fowler, M. Mariantoni, J. M. Martinis, and A. N. Cleland, Surface Codes: Towards Practical Large-Scale Quantum Computation, Phys. Rev. A 86, 032324 (2012).

[37] J. M. Taylor, H. A. Engel, W. Dur, A. Yacoby, C. M. Marcus, P. Zoller, and M. D. Lukin, Fault-Tolerant Architecture for Quantum Computation Using Electrically Controlled Semiconductor Spins, Nat. Phys. 1, 177 (2005).

[38] S. C. Benjamin, B. W. Lovett, and J. M. Smith, Prospects for Measurement-Based Quantum Computing with Solid State Spins, Laser Photonics Rev. 3, 556 (2009).

[39] N. C. Jones, R. Van Meter, A. G. Fowler, P. L. McMahon, J. Kim, T. D. Ladd, and Y. Yamamoto, Layered Architecture for Quantum Computing, Phys. Rev. X 2, 031007 (2012).

[40] N. H. Nickerson, Y. Li, and S. C. Benjamin, Topological Quantum Computing with a Very Noisy Network and Local Error Rates Approaching One Percent, Nat. Commun. 4, 1756 (2013).

[41] Y. Li, S. D. Barrett, T. M. Stace, and S. C. Benjamin, Fault Tolerant Quantum Computation with Nondeterministic Gates, Phys. Rev. Lett. 105, 250502 (2010)

[42] L. M. Duan and H. J. Kimble, Scalable Photonic Quantum Computation through Cavity-Assisted Interactions, Phys. Rev. Lett. 92, 127902 (2004).

[43] W. Yao, R. Liu, and L. J. Sham, Nanodot-Cavity Electrodynamics and Photon Entanglement, Phys. Rev. Lett. 92, 217402 (2004).

[44] E. Waks and J. Vuckovic, Dipole Induced Transparency in Drop-Filter Cavity-Waveguide Systems, Phys. Rev. Lett. 96, 153601 (2006).

[45] S. D. Barrett and P. Kok, Efficient High-Fidelity Quantum Computation Using Matter Qubits and Linear Optics, Phys. Rev. A 71, 060310(R) (2005).

[46] C. Y. Hu, A. Young, J. L. O’Brien, W. J. Munro, and J. G. Rarity, Giant Optical Faraday Rotation Induced by a Single-Electron Spin in a Quantum Dot: Applications to Entangling Remote Spins via a Single Photon, Phys. Rev. B 78, 085307 (2008).

[47] C. Y. Hu, W. J. Munro, J. L. OBrien, and J. G. Rarity, Proposed Entanglement Beam Splitter Using a QuantumDot Spin in a Double-Sided Optical Microcavity, Phys. Rev. B 80, 205326 (2009).

[48] C. Bonato, F. Haupt, S. S. R. Oemrawsingh, J. Gudat, D. Ding, M. P. van Exter, and D. Bouwmeester, CNOT and Bell-State Analysis in the Weak-Coupling Cavity QED Regime, Phys. Rev. Lett. 104, 160503 (2010).

[49] A. B. Young, C. Y. Hu, and J. G. Rarity, Generating Entanglement with Low-Q-Factor Microcavities., Phys. Rev. A 87, 012332 (2013).

[50] Y. Li, S. Barrett, T. Stace, and S. Benjamin, Long Range Failure-Tolerant Entanglement Distribution, New J. Phys. 15, 023012 (2013).
[51] A. Stute, B. Casabone, B. Brandstätter, K. Friebe, T. E. Northup, and R. Blatt, Quantum-State Transfer from an Ion to a Photon, Nat. Photonics 7, 219 (2013).

[52] J. Volz, R. Gehr, G. Dubois, J. Estéve, and J. Reichel, Measurement of the Internal State of a Single Atom without Energy Exchange, Nature (London) 475, 210 (2011).

[53] K. De Greve, L. Yu, P. L. McMahon, J. S. Pelc, C. M. Natarajan, N. Y. Kim, E. Abe, S. Maier, C. Schneider, M. Kamp, S. Höfling, R. H. Hadfieeld, A. Forchel, M. M. Fejer, and Y. Yamamoto, Quantum-Dot Spin-Photon Entanglement via Frequency Downconversion to Telecom Wavelength, Nature (London) 491, 421 (2012).

[54] W. B. Gao, P. Fallahi, E. Togan, J. Miguel-Sanchez, and A. Imamoglu, Observation of Entanglement between a Quantum Dot Spin and a Single Photon, Nature (London) 491, 426 (2012).

[55] J. R. Schaibley, A. P. Burgers, G. A. McCracken, L. M. Duan, P. R. Berman, D. G. Steel, A. S. Bracker, D. Gammon, and L.J. Sham, Demonstration of Quantum Entanglement between a Single Electron Spin Confined to an InAs Quantum Dot and a Photon, Phys. Rev. Lett. 110, 167401 (2013).

[56] C. Santori, D. Fattal, and Y. Yamamoto, Single-Photon Devices and Applications (Wiley, New York, 2010).

[57] H. J. Kimble, Strong Interactions of Single Atoms and Photons in Cavity QED, Phys. Scr. T76, 127 (1998).

[58] See Supplemental Material at http://link.aps.org/ supplemental/10.1103/PhysRevX.4.031022 for full details of the diamond module, its fault tolerant operation and associated error rates.

[59] W. J. Munro, K. A. Harrison, A. M. Stephens, S. J. Devitt, and K. Nemoto, From Quantum Multiplexing to HighPerformance Quantum Networking, Nat. Photonics 4, 792 (2010).

[60] It is critical when using the ${ }^{15} \mathrm{~N}$ nuclear spin for information storage that the electron spin is not optically excited. Optical excitation of the electron spin can lead to enhanced decay of the nuclear spin.

[61] Kai-Mei C. Fu, C. Santori, P. E. Barclay, L. J. Rogers, N. B. Manson, and R. G. Beausoleil, Observation of the Dynamic Jahn-Teller Effect in the Excited States of Nitrogen-Vacancy Centers in Diamond, Phys. Rev. Lett. 103, 256404 (2009).

[62] A. Batalov, V. Jacques, F. Kaiser, P. Siyushev, P. Neumann, L. J. Rogers, R. L. McMurtrie, N. B. Manson, F. Jelezko, and J. Wrachtrup, Low Temperature Studies of the ExcitedState Structure of Negatively Charged Nitrogen-Vacancy Color Centers in Diamond, Phys. Rev. Lett. 102, 195506 (2009).

[63] J. R. Maze, A. Gali, E. Togan, Y. Chu, A. Trifonov, E. Kaxiras, and M. D. Lukin, Properties of Nitrogen-Vacancy Centers in Diamond: The Group Theoretic Approach, New J. Phys. 13, 025025 (2011).

[64] P. Tamarat, N. B. Manson, J. P. Harrison, R. L. McMurtrie, A. Nizovtsev, C. Santori, R. G. Beausoleil, P. Neumann, T. Gaebel, F. Jelezko, P. Hemmer, and J. Wrachtrup, Spin-Flip and Spin-Conserving Optical Transitions of the NitrogenVacancy Centre in Diamond, New J. Phys. 10, 045004 (2008).

[65] H. Kaupp, C. Deutsch, H.-C. Chang, J. Reichel, T. W. Hansch, and D. Hunger, Scaling Laws of the Cavity 
Enhancement for Nitrogen-Vacancy Centers in Diamond, Phys. Rev. A 88, 053812 (2013).

[66] G. W. Biedermann, F. M. Benito, K. M. Fortier, D. L. Stick, T. K. Loyd, P. D. D. Schwindt, C. Y. Nakakura, R. L. Jarecki, Jr., and M. G. Blain, Ultrasmooth Microfabricated Mirrors for Quantum Information, Appl. Phys. Lett. 97, 181110 (2010).

[67] R. Raussendorf and H. J. Briegel, A One-Way Quantum Computer, Phys. Rev. Lett. 86, 5188 (2001).

[68] S. Felton, A. M. Edmonds, M. E. Newton, P. M. Martineau, D. Fisher, D. J. Twitchen, and J. M. Baker, Hyperfine Interaction in the Ground State of the Negatively Charged Nitrogen Vacancy Center in Diamond, Phys. Rev. B 79, 075203 (2009).

[69] A. Y. Kitaev, Fault-Tolerant Quantum Computation by Anyons, Ann. Phys. (Amsterdam) 303, 2 (2003).

[70] E. Dennis, A. Kitaev, A. Landahl, and J. Preskill, Topological Quantum Memory, J. Math. Phys. (N.Y.) 43, 4452 (2002).

[71] R. Stock and D. F. V. James, Scalable, High-Speed Measurement-Based Quantum Computer Using Trapped Ions, Phys. Rev. Lett. 102, 170501 (2009).

[72] S. J. Devitt, A. G. Fowler, A. M. Stephens, A. D. Greentree, L. C. L. Hollenberg, W. J. Munro, and K. Nemoto, Architectural Design for a Topological Cluster State Quantum Computer, New J. Phys. 11, 083032 (2009).

[73] S. J. Devitt, A. M Stephens, W. J. Munro, and K. Nemoto, Requirements for Fault-Tolerant Factoring on an AtomOptics Quantum Computer, Nat. Commun. 4, 2524 (2013).
[74] A. M. Stephens, Fault-Tolerant Thresholds for Quantum Error Correction with the Surface Code, Phys. Rev. A 89, 022321 (2014).

[75] G. Balasubramanian, P. Neumann, D. Twitchen, M. Markham, R. Kolesov, N. Mizuochi, J. Isoya, J. Achard, J. Beck, J. Tissler, V. Jacques, P. R. Hemmer, F. Jelezko, and J. Wrachtrup, Ultralong Spin Coherence Time in Isotopically Engineered Diamond, Nat. Mater. 8, 383 (2009).

[76] T. Ishikawa, K.-M. C. Fu, C. Santori, V. M. Acosta, R. G. Beausoleil, H. Watanabe, S. Shikata, and K. M. Itoh, Optical and Spin Coherence Properties of NitrogenVacancy Centers Placed in a $100 \mathrm{~nm}$ Thick Isotopically Purified Diamond Layer, Nano Lett. 12, 2083 (2012).

[77] K. Fang, V. M. Acosta, C. Santori, Z. Huang, K. M. Itoh, H. Watanabe, S. Shikata, and R. G. Beausoleil, High-Sensitivity Magnetometry Based on Quantum Beats in Diamond Nitrogen-Vacancy Centers, Phys. Rev. Lett. 110, 130802 (2013).

[78] A. M. Stephens, J. Huang, K. Nemoto, and W. J. Munro, Hybrid-Systems Approach to Fault-Tolerant Quantum Communication, Phys. Rev. A 87, 052333 (2013).

[79] X. F. He, N. B. Manson, and P. T. H. Fisk, Paramagnetic Resonance of Photoexcited N-V Defects in Diamond. II. Hyperfine Interaction with the ${ }^{14} \mathrm{~N}$ Nucleus, Phys. Rev. B 47, 8816 (1993).

[80] N. B. Manson, J. P. Harrison, and M. J. Sellars, NitrogenVacancy Center in Diamond: Model of the Electronic Structure and Associated Dynamics, Phys. Rev. B 74, 104303 (2006). 